\title{
Towards a Taxonomy for the Development of Older Adults Healthcare Applications
}

\author{
Ítalo Linhares de Araújo, Evilasio Costa Junior, Paulo Duarte, Ismayle de Sousa Santos, Pedro Almir Martins Oliveira, \\ Cícero Marcelo Oliveira Mendes, and Rossana Maria de Castro Andrade \\ Federal University of Ceará - Brazil \\ \{italoaraujo, evilasiojunior, pauloduarte, ismaylesantos, pedromartins, \\ ciceromendes\}@great.ufc.br, rossana@ufc.br \\ Ivana Cristina de Holanda Cunha Barreto, and Luiz Odorico Monteiro de Andrade \\ Fundação Oswaldo Cruz - Brazil \\ \{ivana.barreto, odorico.monteiro\} @ fiocruz.br
}

\begin{abstract}
The world population is aging and this motivates the development of diferent software solutions focused on the older adults' health. In the literature, however, there is a lack of a organization of the information used by these software. To address this gap, we present in this paper a taxonomy to support the development of software applications dealing with the older adults' health. This taxonomy is composed by 87 characteristics and 21 categories that organize the information that should be handled by this kind of application. The assessment of our taxonomy was performed with a group of five ICT experts with experience in digital healthcare and two healthcare professionals that work with older adutls. From this evaluation, we improve the taxonomy according to the inter-rater agreement among the experts. As a result, our taxonomy may be used to guide the development of healthcare applications for older adults.
\end{abstract}

\section{Introduction}

The aging population is a phenomenon that is occurring all over the world. This circumstance increases the costs of healthcare for older adults and generates demand for solutions that allow a better monitoring of their health [1]. Moreover, according to the World Health Organization (WHO) [2], the creation of new applications aimed at the health of the older adults is a strategic action to deal with the aging of the population.

The development of applications for monitoring and care of the health of older adults has then become one of the main focus of research in the area of digital health in recent years [3]. However, in the literature, there is a gap of the information types organization that can be used in applications for the older adults. These data can be identified through dialogue with health experts or using instruments for the collection of elderly health data already evaluated by these professionals. Hence, this work has as goal:

- To define a taxonomy to guide the development of healthcare applications with a focus in older adults.

Thus, we present a comprehensive taxonomy for healthcare applications targeted to older adults. Starting from the Brazilian Health Handbook for the Elderly [4], we follow the taxonomy development method by Nickerson et al. [5] to extract and expand the information obtained in the handbook. As a result, we generate a taxonomy composed of a total of eighty-seven characteristics organized into twenty-one categories. These categories and characteristics encompass an extensive set of health information related to older people that must be handled by the applications so that they can perform the specific actions of each type of system shown in the taxonomy.

Initially, our focus encompassed seven types of healthcare applications for older adults (home monitoring healthcare system, self-care application, epidemiological clinic system, medical normative system, and medical governance). Because the types of applications have differences in purpose and end-user, not all characteristics or categories proposed are essential for each type. To define what should be essential for each type of health application, we validated the taxonomy with two healthcare professionals and five Information and Communications Technology (ICT) specialists with experience in digital healthcare. 
The remainder of the paper is organized as follows: In Section 2, we present the state-of-the-art for taxonomies focused on digital health systems. Our taxonomy development methodology is presented in Section 3. In Section 4, we present the proposed taxonomy, show the validation performed, and discuss its results. We concluded the paper in Section 5 with our final considerations.

\section{Related Work}

We identified in literature work related to taxonomies for digital health systems [6][7][5][8][9][10] and other context-aware applications [11][12] that were the basis for our research.

In [6], the authors propose a taxonomy for mobile health applications in South Africa. This taxonomy categorizes various dimensions of mHealth applications and provides a standard for new applications. [7] presents a methodology for applying gamification elements in health apps following the method proposed in [5]. The authors of [8] propose a framework of building simulations for healthcare applications and a taxonomy for supporting this framework. This framework and taxonomy contain four types of simulations related to the simulation experience: computer-based simulation, simulated patient, simulated clinical immersion, and procedural simulation. In [9], the authors propose a taxonomy that helps in evaluating mobile apps for eHealth in terms of privacy and security.

In [10], the authors propose a taxonomy created with basis in a literature survey for mHealth applications, using the qualitative research coding approach [13] and also the methodology presents in [5]. This taxonomy is divided into three dimensions (Medical Use Cases, Technical Modalities, and Policy Considerations), and each dimension contains a set of categories. In the Medical Use Cases Dimension are considered characteristics specifics for healthcare applications, as the point of care diagnostic, wellness, education and reference, efficiency and productivity, the patient monitoring, compliance, behavior modification, and environmental monitoring. The Technical Modalities dimension represents technical information about mHealth Applications, as device types, interfaces, OS types, and features. Finally, the Policy Consideration dimension highlights information about regulation, data management, and security.

Polash et al. (2015) [11] presents a survey on the state-of-the-art of cloud computing, and from it, they created a taxonomy of cloud computing focusing on three main aspects 1) Concepts, 2) Performance, and 3) Security. Lastly, [12] introduces and tests the applicability of a systematic taxonomy development approach. Besides, the paper presents the process of developing a taxonomy that is based on the consensus shared among the participants of an assessment.

Like in these studies, in our taxonomy, we also present information for help developers to create healthcare applications, but we focus on the applications for improvement the older adults health. With this approach, we consider the relevant information specific for the older adults care. We also divide the information (or characteristics) in the framework in categories to facilitate the developments of applications, however, in our taxonomy, we do not deal with aspects of security, as in [9] [11] and [10]. It is also important to highlight that for constructing our taxonomy, such as [7] and [10], we use the methodology describes in [5].

\section{Research Approach}

To construct the taxonomy, we started by the analysis of the 4th edition of the Brazilian Health Handbook for the Elderly [4], which includes several relevant aspects to evaluate the health of the elderly. The booklet was created in 2006 by Brazil's Ministry of Health in partnership with the Coordination of Health of the Elderly Person (COSAPI) and with the Foundation Oswaldo Cruz (FIOCRUZ), as an instrument to help in the management of the health of the older adults. The material allows the registration and follow-up, for five years, of information such as personal, social, and family data, as well as the health conditions of the elderly and their life habits [14] [15].

In addition to the analysis of the Brazilian Health Handbook for the Elderly, we searched the literature for work that proposed taxonomies for applications in the context of digital health, to support this research and to understand the main aspects covered by these taxonomies, besides selecting a methodology that could guide our taxonomy development process. Figure 1 shows the process followed in this research and its main activities are detailed in the following subsections.

\subsection{Mindmap Development}

To create the mindmap, we start the process of analyzing the information present in the 4th edition of the Brazilian Health Handbook. The first step was the identification of the categories of information. We identify two main categories: (i) personal data of the older adult or the reference person, and (ii) treatment. We consider that these categories are adequate and complete, then we keep them in the mindmap.

In sequence, we observe that these two categories 


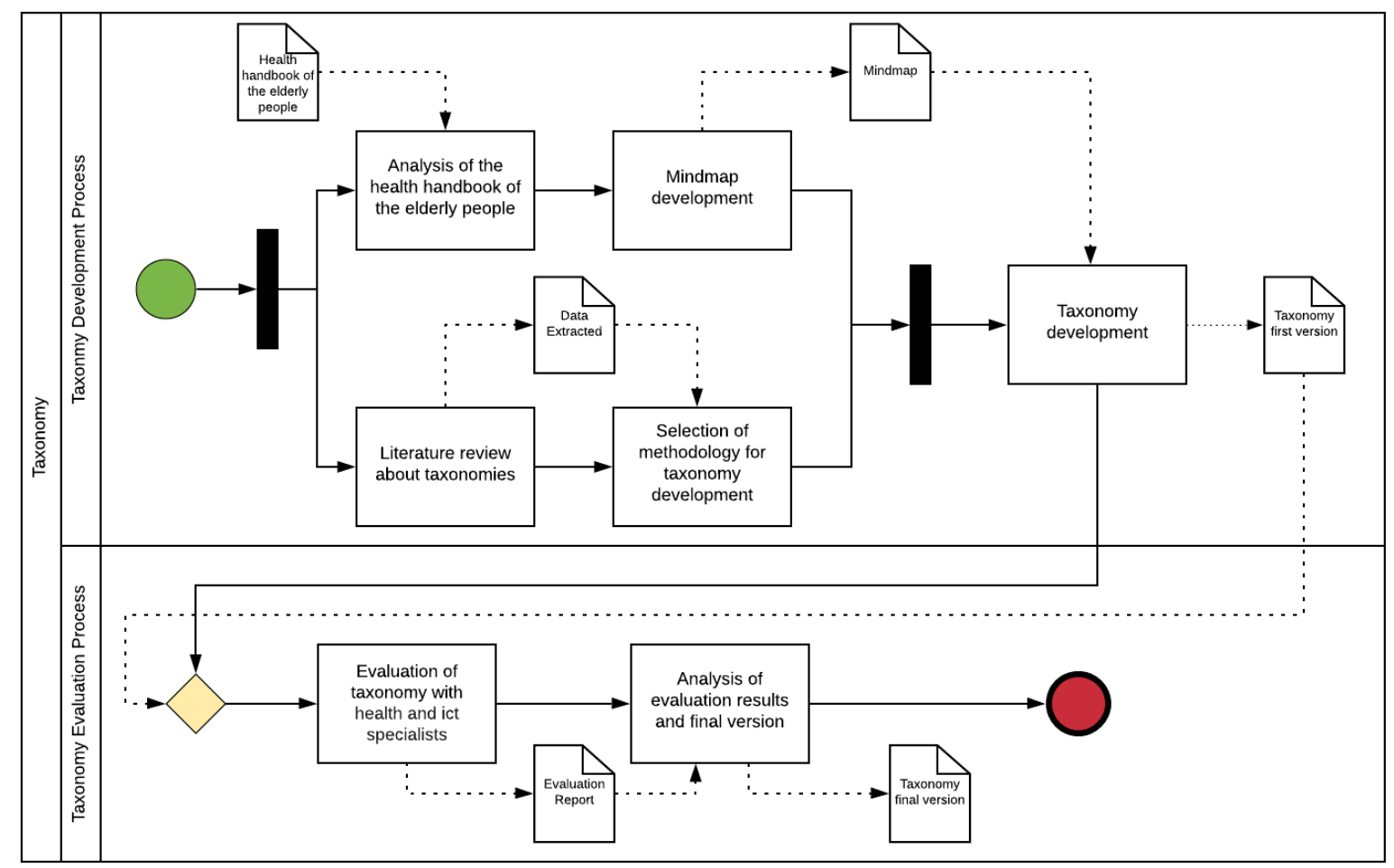

Figure 1. Research process

have subcategories. The first one is the personal data, which can be related to older adult identification, contact information, schooling, information about general health, as blood type, specific health, as allergy or deficiency, and the last one is the birth information. The second is the treatment that deals with diseases and surgeries until ambiental evaluation.

We continue to analyze the handbook, and we note that they are complete in the categories: personal data, data of the reference person, and social-family information. The treatment data are not complete, then we search in other sources, as a prescription, together with our experience to complete the data. An example of this is the medicine with data of the health staff, considering from name until the number of the regional council of the area including the service unit. Another example is the surgery data that we include the data staff.

Moreover, we expand the options that were not clear or were confused. Thus, we reorganize the data to indicate the better corresponding with the reality and thinking in how the users could use the data. For example, we can cite the data related to body mass index, and we classify the result in three categories: low weight, adequate weight, and overweight. Another example is in the medicine, which fields have the same value in the duration and start time, and we consider that is different information, and we split into two fields to better register the information.

To validate the mindmap, we submit the mindmap for four health specialists, all of the different areas. They could analyze the mindmap in two moments: first, for two weeks, they could analyze and make suggestions. In the second moment, a meeting was performed to present the mindmap to solve any doubt or to correct any problem. The final mindmap is visualized in: https://bit.ly/2KSHEjw.

\subsection{Literature Review}

The literature review executed in this research looked for to identify papers that propose taxonomies for applications in the context of digital health. First, we define a search strategy in academic articles databases and relevant conferences and journals (in science computer area). After, we apply the inclusion and exclusion criteria to select the papers more relevant. Then, we extract the data, and we analyze and synthesis of the results. Four researchers executed these activities.

In total, we select 12 articles for complete reading and data extraction. However, before the synthesis, we disregarded four papers published before 2010. We then synthesized the data extracted from the remaining eight 
papers and analyzed the main aspects of the taxonomies presented by these studies and the process followed to construct the taxonomies by them.

Based on these studies, we identified the methodology for developing taxonomies for information systems applications proposed in [5], which was followed by two of the papers [7] [10]. We analyze the quantity and quality of the studies that cite this methodology uses the Google Scholar 1 and with this, we verify that this methodology was used by many papers published in conferences and journals well recognized by the academic community. Then, we did a detailed check of this methodology and chose to use it to guide the development phase of our taxonomy.

We highlight that no other taxonomies development methodology detailed and followed by more than one work that we study during the literature review. However, Although a more comprehensive literature review could indicate other approaches. We believe that the methodology selected is useful for the type of research we propose in our article.

\subsection{Taxonomy Development}

As mentioned before, for developing our taxonomy, we use the methodology proposed in [5]. Figure 2 shows the process of this methodology. Briefly, the methodology proposes an interactive process of taxonomy construction. In each interaction, new elements (characteristics, dimensions, or applications) are added to taxonomy. The process ends when the pre-set stop conditions are reached. Next, we will describe the process of taxonomy construction using this methodology.

3.3.1. Meta-characteristic. First, we defined the following objective: to create a taxonomy to assist the development of digital health applications focused on the older adult, targeting researchers and developers of digital health systems.

With this purpose, we determined as meta-characteristics that define what will have in our taxonomy: common and relevant information to the development of digital health applications focused on the elderly presented in the mindmap.

3.3.2. Ending conditions. As for ending conditions, we select three objective criteria and one subjective. We have the following objective conditions:

- When all objects from the mindmap were verified.

\footnotetext{
${ }^{1}$ https://scholar.google.com
}

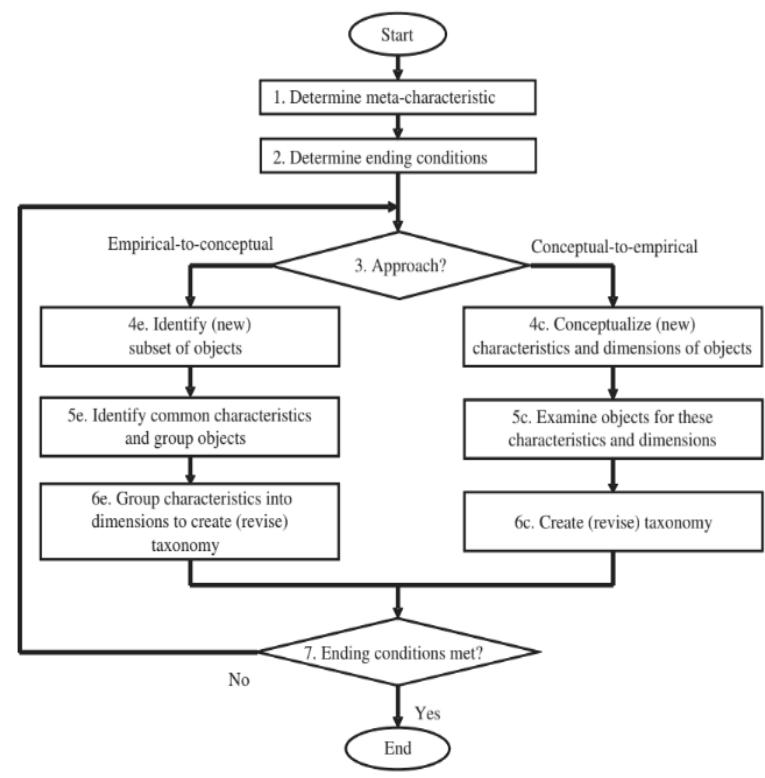

Figure 2. Taxonomy development methodology [5]

- When all dimensions are unique.

- When all the characteristics are unique in each of the dimensions to which they are associated.

And the subjective condition that we have is:

- When the authors of this taxonomy considered all types of applications selected.

3.3.3. Interactions. During the taxonomy development process, we performed six interactions. Figure 3 shows the categories (or dimensions) and applications added in each interaction.

Initially, the taxonomy was thought to work with two types of applications: applications to assist older adults to care [3] and applications of medical treatment and follow-up [16] [1]. Considering these applications and based on the mindmap, we performed five interactions following the steps of the "Empirical-to-Conceptual" methodology process. In each interaction, we added to taxonomy a set of categories and information (or characteristics) related to these categories.

In the last interaction, we follow once again the steps of the "Empirical-to-Conceptual" methodology process. In this interaction, we verified in the literature other types of health applications aimed at the care of the elderly, and we added five types of applications: Home monitoring healthcare system [17] [3], Self-care application [18], Epidemiological Clinic System [19], Medical Normative System [19], and Medical Governance [19] [1]. 


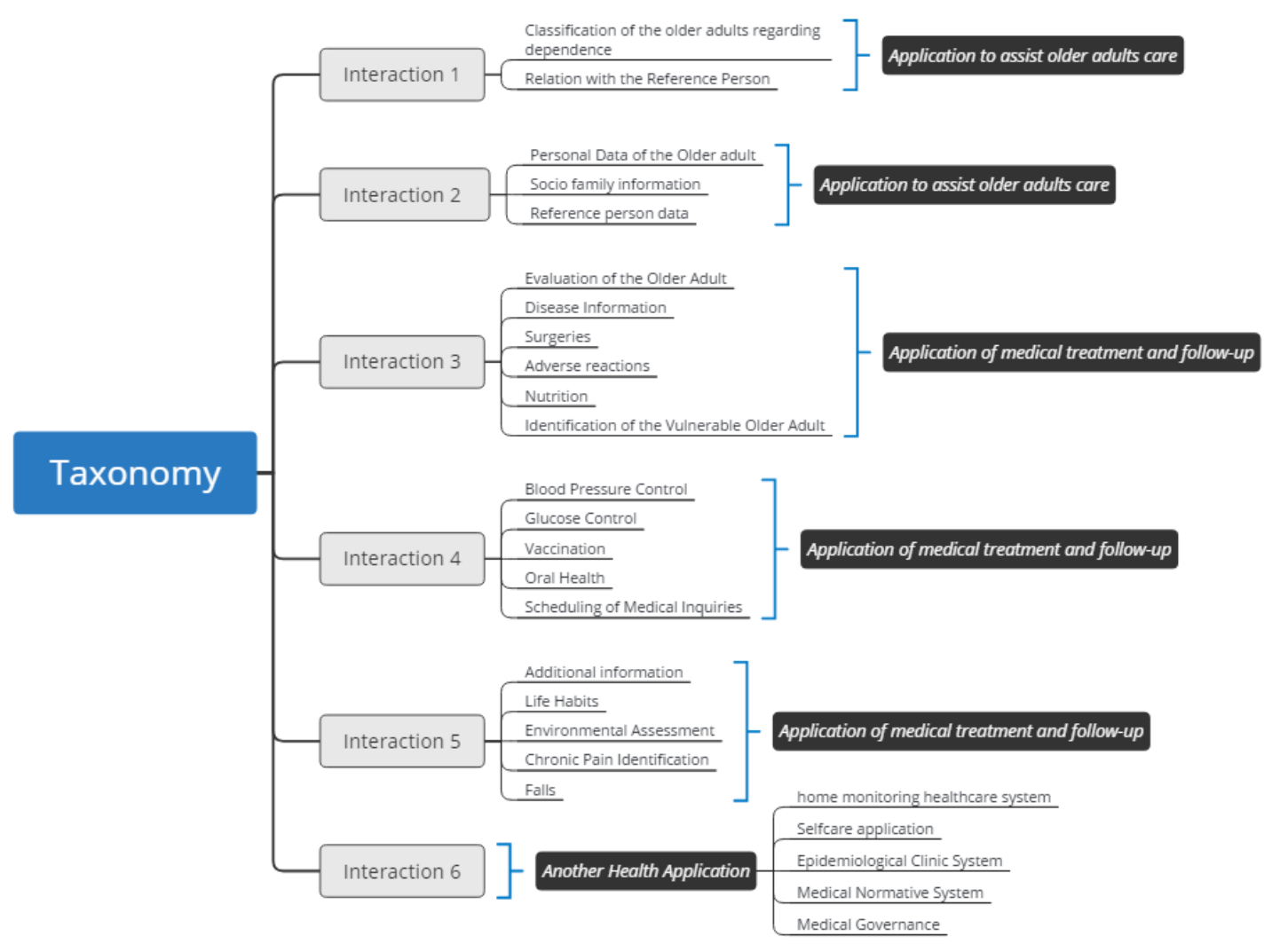

Figure 3. Taxonomy development interactions

Each information, related to each type of application, can assume one of three values: Essential, Optional, or None. When a characteristic is considered Essential, it must be present in the app, if it is Optional, it may or may not be in the app, and when it is None, it does not add value to app and soon should not be in it. Section 4 will detail the types of applications, categories, and information present in the taxonomy.

At the end of the execution of the methodology, we obtained the first version of the taxonomy, which we submitted for the evaluation of specialists.

\subsection{Taxonomy Evaluation}

To validate the information, categories, and applications, in addition to the values of each information, an evaluation was performed with health and ICT specialists. The taxonomy evaluation process is illustrated in Figure 4

First, we created a spreadsheet with our taxonomy, in which the columns contain the application types, and the lines contain the information divided into the categories shown in Figure 3. We also prepared a document describing each element of the taxonomy and a profile form. Then we send the documents to specialists analysis.

Each specialist answered the profile form and analyzed the spreadsheet to give feedback in the elements of the taxonomy and indicated the values (Essential, Optional, or None) that they considerate correct for each information.

We work with five experts from the ICT area and two from the health area. All ICT specialists have a master's degree in Computer Science, and four of them are doctoral students. Besides, all five have experience in the area of health systems development and research, ranging from one to five years of experience. Concerning health specialists, we consulted a gerontologist, and a general clinic, all with more than nine years of experience in their areas.

After obtaining the answers and considerations of the specialists, they were analyzed to verify the difference between the response use the radar charts. Furthermore, to achieve the current version of the taxonomy, we use the mode statistical analysis to identify the answer most cited by the evaluators. We perform this with the data of ICT, health, and both evaluators and the final summary is presented in the next 
Table 1. Taxonomy values for three categories. E - Essential, O - Optional, N - None

\begin{tabular}{|c|c|c|c|c|c|c|c|c|c|c|c|c|c|}
\hline & \multicolumn{2}{|c|}{$\begin{array}{c}\text { Classification of the } \\
\text { older adults regarding } \\
\text { dependence }\end{array}$} & \multicolumn{3}{|c|}{$\begin{array}{l}\text { Relation with the } \\
\text { reference person }\end{array}$} & \multicolumn{5}{|c|}{ Disease Information } & \multicolumn{3}{|c|}{$\begin{array}{l}\text { Identification of the } \\
\text { Vulnerable Older Adult }\end{array}$} \\
\hline & 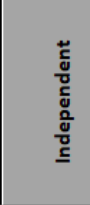 & 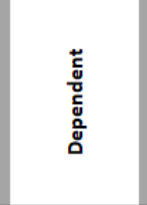 & 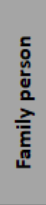 & 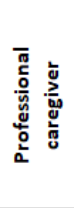 & $\begin{array}{l}\text { 오 } \\
\text { ه̊ }\end{array}$ & 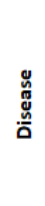 & 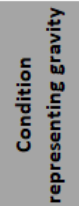 & 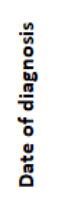 & 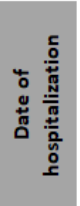 & 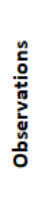 & 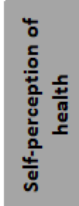 & 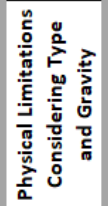 & 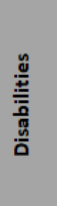 \\
\hline Application to assist older adult care & & $\mathrm{E}$ & $\mathrm{E}$ & $\mathrm{E}$ & $\mathrm{E}$ & $\mathrm{E}$ & $\mathrm{E}$ & $\mathrm{E}$ & $\mathrm{E}$ & $\mathrm{E}$ & $\mathrm{E}$ & $\mathrm{E}$ & $\mathrm{E}$ \\
\hline $\begin{array}{l}\text { Applications of medical treatment and } \\
\text { follow-up }\end{array}$ & & E & $\mathrm{E}$ & E & E & $\mathbf{E}$ & E & $\mathbf{E}$ & $\mathrm{O}$ & $\mathrm{O}$ & E & E & $\mathbf{E}$ \\
\hline Home monitoring healthcare systems & & E & $\mathrm{E}$ & $\mathrm{O}$ & $\mathrm{E}$ & $\mathrm{E}$ & $\mathrm{E}$ & $\mathrm{N}$ & $\mathrm{N}$ & $\mathrm{E}$ & $\mathrm{E}$ & $\mathrm{E}$ & $\mathrm{E}$ \\
\hline Selfcare application & & E & $\mathrm{N}$ & E & $\mathrm{E}$ & $\mathrm{E}$ & $\mathrm{E}$ & $\mathrm{N}$ & $\mathrm{E}$ & $\mathrm{E}$ & $\mathrm{E}$ & $\mathrm{E}$ & $\mathrm{E}$ \\
\hline Epidemiological clinic systems & & E & $\mathrm{E}$ & 0 & $\mathrm{E}$ & $\mathrm{E}$ & E & $\mathrm{E}$ & E & $\mathrm{O}$ & $\mathrm{O}$ & $\mathrm{E}$ & $\mathrm{E}$ \\
\hline Medical normative systems & & E & $\mathrm{O}$ & E & $\mathrm{E}$ & $\mathrm{E}$ & $\mathrm{E}$ & $\mathrm{N}$ & $\mathrm{N}$ & $\mathrm{E}$ & $\mathrm{N}$ & $\mathrm{E}$ & $\mathrm{E}$ \\
\hline Medical governance applications & & E & $\mathrm{E}$ & $\mathrm{N}$ & $\mathrm{E}$ & $\mathrm{E}$ & E & $\mathrm{E}$ & E & $\mathrm{N}$ & E & E & $\mathrm{E}$ \\
\hline
\end{tabular}

section.

\section{Taxonomy to Older Adults Healthcare Applications}

Our taxonomy contains eighty-seven characteristics into twenty-one categories. These characteristics represent information that should have, or not, in each type of application. In our taxonomy, we work with seven types of applications related the older adults care.

\subsection{Types of applications}

Application to assist older adult care corresponds to applications aimed at caregivers (family or professional) of older adults. Applications of medical treatment and follow-up are applications focused on monitoring medical treatment. Home monitoring healthcare systems are applications aimed at monitoring the health of the elderly within a specific environment (e.g., their home). Selfcare application represents types of applications focused on the independent elderly capable of performing self-care actions.

Epidemiological clinic systems are applications that aim to assure the health manager knowledge about the health-disease process, knowing that health takes into account an entire social, biological, economic, and environmental context of the individual. Medical normative systems assist law enforcement actions by health managers. According to [19], these systems aiming at generating standards that can give consistency, concreteness, and legal certainty to the health systems. Finally, Medical governance applications are related to the whole process of management of inputs, personnel, infrastructure, organizational structure, management forms, which will support the health system. They include administrative management and shared management.

\subsection{Categories and characteristics}

Table 1 shows the values of the current version of our taxonomy information for three categories. Lines present the types of applications and columns contains categories and characteristics. Following we detailed all categories and characteristics. The complete taxonomy is available in: https://bit.ly/2KSHEjw

1. Classification of the older adults regarding dependence. Identifies the type (s) of the older adults to which the application is directed. The characteristics related to this category are: Dependent or Independent, an older adult who needs help to perform self-care activities;

2. Relation with the reference person. Identifies what kind of relationship exists between the older adult and the responsible person of reference if something can happen to the older adult. The characteristics related to this category are: Family person, a relative of the older adult; Professional caregiver, a professional caregiver of older adults; Doctor, a doctor of older adults.

3. Personal data of the older adult. Identifies personal data of the older adult. The characteristics related to this category are: Identification number, identification number of the older adult in the federal health system; Name), name of the older adult; Date of birth, date of birth of the older adult; Gender, gender of the older adult; Nationality, nationality of the older adult; Schooling, schooling of the older 


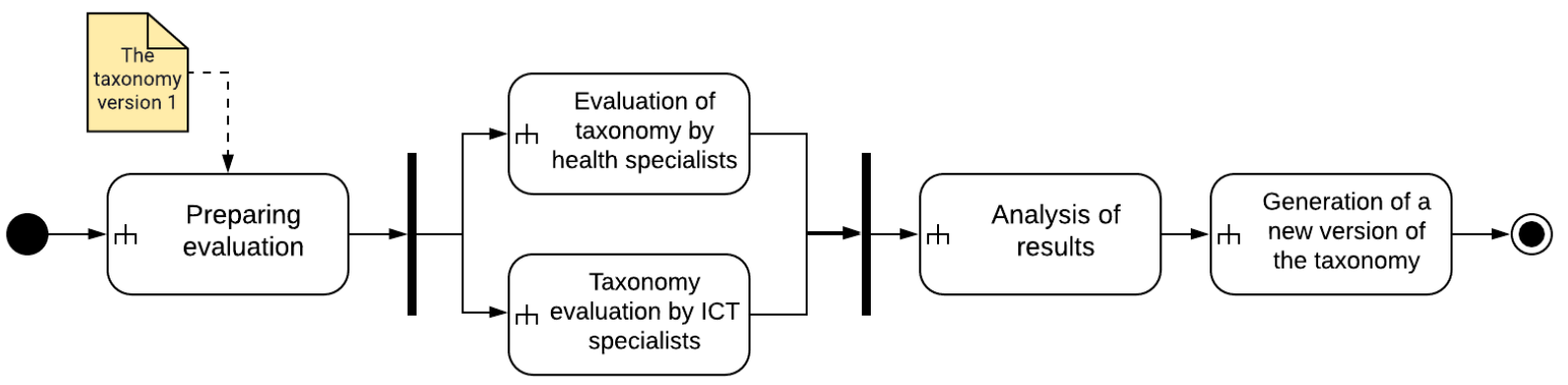

Figure 4. Taxonomy evaluation process

adult; Ethnicity, ethnicity of the older adult; Religion, religion of the older adult; Conjugal situation, conjugal situation of the older adult; Allergy, allergy situation of the older adult; Blood type, blood type of the older adult; Deficiency, older adult has any deficiency; Contact information, contact information of the older adult (e.g., phone number or email).

4. Socio family information. Information about the elderly family. The characteristics related to this category are: Who does the older adult live with?, the elderly live with a relative, a caregiver or only; Performed activities, the older adult perform activities continuously?; Ease of access to transport Does the older adult have ease of access to transport?; Work actively Does the older adult Work actively?; Source of income, what is the source of income for the elderly?

5. Reference person data. Identifies personal data of the reference person. The characteristics related to this category are: Name, name of the reference person; Date of birth , date of birth of the reference person; Contact information contact information of the reference person (e.g., phone number or email); Living with the older adult, the reference person living with the older adult?

6. Evaluation of the older adult. Information on medications used by the older adult. The characteristics related to this category are: Prescription of the medication, medications prescribed by a physician; Health professional prescribed the medicine, identification of the doctor that prescribed the medication; Polypharmacy, Do the older adults have polypharmacy?

7. Disease information. Identifies disease information. The characteristics related to this category are: Disease, diseases that the elderly have; Condition representing gravity, How dangerous is the health situation of the elderly?; Date of diagnosis, The date the diagnosis was reported; Date of hospitalization, The length of time that the older adult was hospitalized; Observations other observations on the disease.

8. Surgeries. Information on surgeries. The characteristics related to this category are: Injured body part, part of the body on which the surgery was performed; Date of surgery, execution date of surgery; Healthcare professional who performed the surgery, identification of healthcare professional who performed the surgery; Observation, other observations on the surgery.

9. Adverse reactions. Information on adverse drug reactions. The characteristics related to this category are: Medication, identification of the medication; Date of the reaction was identified, date of the adverse reaction was identified; Symptoms, symptoms related to the adverse reaction.

10. Nutrition. Information on the older adults diet. The characteristics related to this category are: Feeding, feeding routine; Anthropometric data, information on anthropometry of the older adult.

11. Identification of the vulnerable older adult. Identification of vulnerabilities in the older adult. The characteristics related to this category are: Self-perception of health, how the older adult perceive your health; Physical Limitations Considering Type and Gravity, Does the older adult have physical limitations?; Disabilities, Does the older adult have disabilities?

12. Blood pressure control. Control of blood pressure. The characteristics related to this category are: Date, date of the blood pressure measurement; Blood pressure, value of the blood pressure measurement.

13. Glucose control. Information on blood glucose control. The characteristics related to this category are: Date, date of the glucose measurement; Glycemia, value of the glucose measurement.

14. Vaccination. Vaccination Information. The characteristics related to this category are: Vaccine, identification of the vaccine; Date, date of the vaccination; Batch, vaccine batch.

15. Oral health. Information on the oral health of the older adult. The characteristics related to this category are: Smoker, does the older adult is a 
smoker?; Do you often drink alcohol?, does the older adult often drink alcohol?; Mucosa Injury or Mucosa Alteration, does the older adult have a mucosa Injury or mucosa alteration?; Dental cavity, does the older adult have a dental cavity?; Periodontal Disease (Bacterial plaque, plaque retention, peritonitis or gingivitis), does the older adult have periodontal disease?; Dental prosthesis, does the older adult use a dental prosthesis?; Periodic clinical follow-up, does the older adult have a periodic clinical follow-up?.

16. Scheduling of medical inquiries. Information about the schedule of medical consultations of the older adult. The characteristics related to this category are: Date/Hour/Local, date, hour and local of the consultation; Consultation/Examination, what were the results of the consultation?; Professional Name, identification of the medical professional name.

17. Additional information. Additional health information and follow-up daily activities of the older adult. The characteristics related to this category are: External perception of forgetfulness, What is the perception of the older adult about forgetfulness?; Impediments caused by forgetfulness, what are the impediments caused by forgetfulness?; Feeling of Despondency, Does the older adult have a feeling of despondency? ; Loss of interest in activities, Did the older adult loss of the interest in any activities?

18. Life habits. Daily habits of the older adult. The characteristics related to this category are: Consume alcoholic beverage, Does the older adult consume alcoholic beverage?; Alcohol consumption problems, Does the older adult have an alcohol consumption problems?; Smoking Status, What is the smoking status of the older adult?; Physical Activity Practice, Does the older adult practice physical activities? Which are?; Practice Leisure Activities, Does the older adult practice leisure activities? Which are?; Frequency in centers/clubs/social groups, What is the frequency of the older adult in centers/clubs/social groups?

19. Environmental assessment. Information about the environment where the older adult live. The characteristics related to this category are: Existence of impediments of locomotion, does the older adult has any impediment of locomotion in the environment?; Existence of locomotion aids, does the older adult use locomotion aids (locomotion subsidy) in the environment?; Lighting, is the lighting in the environment adequate? Is there enough light for the elderly to visualize the environment?; Kitchen and bedroom, have health security items in Kitchen and bedroom?; Bathroom, have health security items in the bathroom?; Stairs, have health security items in the ladder?

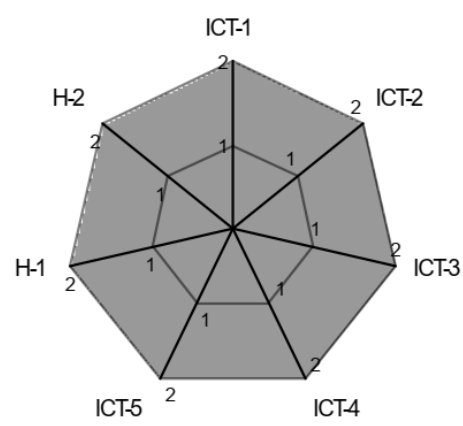

Figure 5. Radar chart for the characteristic "Dependent/Independent" of the category Classification of the older adults regarding dependence.

20. Chronic pain identification. Information on chronic pain. The characteristics related to this category are: Duration, duration of the pain; Sensation, what is the sensation of the older adult related to this pain?; Local, what is the location of the pain?; Intensity, what is the intensity of the pain?

21. Falls. Information on the occurrence of falls. The characteristics related to this category are: Date, date of the fall; Local, local of the fall; Fracture, description of the fracture related to the fall; Consequences, health consequences of falling.

\subsection{Evaluation Results}

With the results of the evaluation done with the specialists of the development of health systems and health professionals, we arrive at the current version of the taxonomy, considering the mode statistical analysis.

When we use the mode statistical analysis, we observe a problem with the responses of some professionals when they disagreed, or when there are more than two values for mode. For example, there are some results that two professionals answered "Essential" and two "Optional". To solve this, we decide to use the answer with the value "Essential" in comparison with the others and the value "Optional" when the conflict is with "None".

We also did a field-by-field analysis for each type of application. For this, we generated radar charts that can be seen in Figures 5 and 6 . In the charts, ICT represents the ICT professionals, $\mathrm{H}$ are the health professionals. And the values 0, 1 and 2 corresponding to "Essential", "Optional", and "None" respectively.

In Figure 5. $100 \%$ of the specialists agreed that characteristic "Dependent/Independent" is essential for applications to assist older adults care. However, in Figure 6, for the same type of app, we can observe 


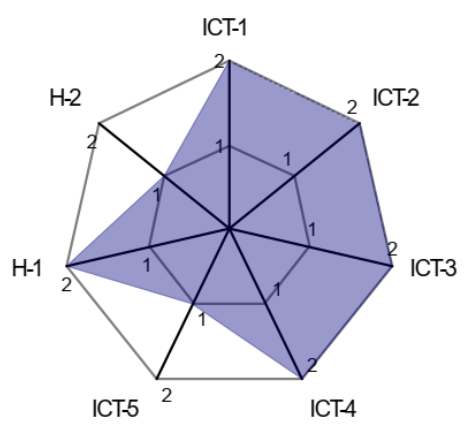

Figure 6. Radar chart for the characteristic "Date of hospitalization" of the category Disease Information.

that two experts considered the characteristic "Date of hospitalization" as optional, and five experts considered it essential. Note that in Table 1, both characteristics have a value essential, because most of specialists chose this value for them.

Aiming to achieve a detailed analysis of the radar charts as well as full access to the current taxonomy version, we have made some files available in a public Google Drive folder that can be accessed by https://bit.ly/2KSHEjw. The taxonomy is available as a table in the Taxonomy.xlsx file. All radar charts - generated using the OriginPro Data Analysis and Graphing Software v9.1 - are in the Taxonomy_RadarCharts.pdf file. With this file, it is possible to observe the agreement among the experts for all 87 taxonomy characteristics. Finally, we also provide the mindmap discussed in section 3.1 in the Mindmap_olderadult.pdf file.

4.3.1. Discussion We decided to use the radar graph to analyze the agreements and disagreements of each specialist answer. This strategy presented interesting results because it was possible to clearly observe the response behavior, considering the characteristics and types of applications.

The complete analysis of radar graphs showed that the types Medical Normative System and Medical Governance Applications have the most significant divergences between the specialists, with only 5\% of unanimity regarding the characteristics of these applications. This low agreement rate reflects the different perspectives of health specialists and ICT specialists concerning normative and governance health systems. In general, ICT professionals seek to optimize systems using the minimum set of characteristics. The vision of health professionals is more comprehensive

\footnotetext{
${ }^{2}$ OriginPro Website: https://www.originlab.com/Origin
}

and aims to provide as much data as possible.

On the other hand, the specialists had a high agreement rate in more than $70 \%$ of the characteristics of applications to assist older adult care. This behavior is probably because this type of application is more common in the eHealth industry as well as in academic studies.

4.3.2. Limitations and Threats to Validity The main threats to validity are: (i) the number of experts involved in the evaluation; (ii) the application types used in the taxonomy; and (iii) the process used to define the taxonomy.

Regarding the number of experts that assessed the taxonomy, despite only five ICT experts participated in the study, all of they have already worked with healthcare applications. Also, from the health domain, we have only two healthcare professionals in the taxonomy's evaluation. However, the taxonomy was defined based on Brazilian Health Handbook for the Elderly, that was validated by several healthcare professionals.

The application types, in turn, were chosen by in the literature research, and, therefore, we believe that these involve a significant number of health applications. Also, even the types list used not being exhaustive, if there are other types not including in the taxonomy proposed, the new type can be compared with the taxonomy to identify the best set of fields to be present.

With regards to the process used, we followed one that is very cited and it was followed in other studies to create several taxonomies in other domains. Also, before we create the taxonomy, we made a mindmap, which was validated with four health professionals.

\section{Conclusions and Future Work}

In this work, we present a taxonomy to older adults healthcare applications. This instrument is aimed at developers and contains a set of information necessary for the development of specific types of health systems focused on the older adults.

The development of the taxonomy was done following an interactive method and based on a mindmap built on the basis of information from Brazilian Health Handbook for the Elderly. Before arriving at the current version, we made also an evaluation of the taxonomy submitting it to the analysis of professionals of the area of Information Technology and Communication and Health professionals with experience in working with the older adults.

The current version of the taxonomy shows 
eighty-seven information organized into twenty-one categories and related to seven types of applications. For each information, the taxonomy identifies if them are essential, optional or if it should not be in each type of app.

As future work, we intend to evaluate the taxonomy with more experts to increase the its reliability also including an evaluation with real healthcare applications. We also intend to do more in-depth research to identify other types of application that can be added to the taxonomy. Finally, we plan to create a framework that contains the taxonomy and from which it would be possible to generate code for the taxonomy's fields depending of the type of application.

\section{Acknowledgments}

The authors would like to thank FIOCRUZ in respect of the financial support for the research (project VPPiS-004-FIO-18), Coordenação de Aperfeiçoamento de Pessoal de Nível Superior - Brasil (CAPES) by the Evilasio Costa Junior PhD scholarship and CNPQ by the Rossana Maria de Castro Andrade DT-2 Productivity Scholarship (Case No. 315543 / 2018-3).

\section{References}

[1] T. D. Robbins, S. N. L. C. Keung, and T. N. Arvanitis, "E-health for active ageing; a systematic review," Maturitas, vol. 114, pp. 34-40, 2018.

[2] WHO, "The global strategy and action plan on ageing and health 2016-2020: towards a world in which everyone can live a long and healthy life," Sixty-ninth World Health Assembly, Geneva, pp. 23-28, 2016.

[3] A. Woll and T. Bratteteig, "A trajectory for technology-supported elderly care work," Computer Supported Cooperative Work (CSCW), vol. 28, no. 1-2, pp. 127-168, 2019.

[4] "Health handbook for the elderly - 4th edition." http://portalarquivos2. saude.gov. $\mathrm{br} / \mathrm{images} / \mathrm{pdf} / 2017 / \mathrm{setembro/27/}$ CADERNETA-PESSOA-IDOSA-2017-Capa-miolo. pdf Accessed: 2019-06-01.

[5] R. C. Nickerson, U. Varshney, and J. Muntermann, "A method for taxonomy development and its application in information systems," European Journal of Information Systems, vol. 22, no. 3, pp. 336-359, 2013.

[6] A. Botha, M. Weiss, and M. Herselman, "Towards a taxonomy of mhealth," in 2018 International Conference on Advances in Big Data, Computing and Data Communication Systems (icABCD), pp. 1-9, IEEE, 2018.

[7] M. Schmidt-Kraepelin, S. Thiebes, M. C. Tran, and A. Sunyaev, "Whats in the game? developing a taxonomy of gamification concepts for health apps," in Proceedings of the 51st Hawaii International Conference on System Sciences, 2018.

[8] G. Chiniara, G. Cole, K. Brisbin, D. Huffman, B. Cragg, M. Lamacchia, D. Norman, and G. W. G. Canadian
Network For Simulation In Healthcare, "Simulation in healthcare: a taxonomy and a conceptual framework for instructional design and media selection," Medical Teacher, vol. 35, no. 8, pp. e1380-e1395, 2013.

[9] M. Plachkinova, S. Andrés, and S. Chatterjee, "A taxonomy of mhealth apps-security and privacy concerns," in 2015 48th Hawaii International Conference on System Sciences, pp. 3187-3196, IEEE, 2015.

[10] P. Olla and C. Shimskey, "mhealth taxonomy: a literature survey of mobile health applications," Health and Technology, vol. 4, no. 4, pp. 299-308, 2015.

[11] F. Polash, A. Abuhussein, and S. Shiva, "A survey of cloud computing taxonomies: Rationale and overview," in The 9th International Conference for Internet Technology and Secured Transactions (ICITST-2014), pp. 459-465, IEEE, 2014.

[12] E. Zainuddin and S. Staples, "Developing a shared taxonomy of workaround behaviors for the information systems field," in 2016 49th Hawaii International Conference on System Sciences (HICSS), pp. 5278-5287, IEEE, 2016.

[13] J. Saldaña, The coding manual for qualitative researchers. Sage, 2015.

[14] "Health care of elderly and aging." http://bvsms . saude.gov.br/bvs/publicacoes/atencao_ saude_pessoa_idosa_envelhecimento_ v12.pdf . Accessed: 2019-06-01.

[15] "Manual for using the health handbook for the elderly." http://bvsms.saude.gov.br/ bvs/publicacoes/manual_utilizacao_ caderneta_pessoa_idosa.pdf. Accessed: 2019-06-01.

[16] O. Banos, M. B. Amin, W. A. Khan, M. Afzal, M. Hussain, B. H. Kang, and S. Lee, "The mining minds digital health and wellness framework," Biomedical engineering online, vol. 15, no. 1, p. 76, 2016.

[17] M. Bajorek and J. Nowak, "The role of a mobile device in a home monitoring healthcare system," in 2011 Federated Conference on Computer Science and Information Systems (FedCSIS), pp. 371-374, IEEE, 2011.

[18] S. Subramaniam, J. S. Dhillon, M. S. Ahmad, T. C. WS LJ, and C. Huang, "Eliciting user requirements to design a prediabetes self-care application: a focus group study with prediabetics and diabetics," Ind J Sci Technol, vol. 9, pp. 1-8, 2016.

[19] M. Oliveira, C. Hairon, O. Andrade, R. Moura, C. Sicotte, J. Denis, S. Fernandes, J. Gensel, J. Bringel, and H. Martin, "A context-aware framework for health care governance decision-making systems: A model based on the brazilian digital tv," in 2010 IEEE International Symposium on" A World of Wireless, Mobile and Multimedia Networks"(WoWMoM), pp. 1-6, IEEE, 2010. 